\title{
Effect of Conductive Filaments on the Electron Emission Properties in Cathodes
}

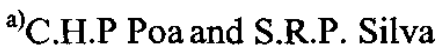 \\ Advanced Technology Institute, School of Electronics and Physical Sciences, University of Surrey, \\ Guildford GU2 7XH, United Kingdom
}

The electron emission process modelled using Fowler-Nordheim theory gives accurate estimates of the emission current when applied to metal. However, when applied to thin film amorphous semiconductors, unexpectedly low effective barriers (Ф) or extremely high field enhancement factors ( $\beta$ ) result. To explain the anomalously high $\beta$ values seen experimentally in amorphous semiconductors two possible sources for field enhancement can be identified; (1) Surface protrusions on the material surface that results in a high geometric enhancement, and (2) Internal field enhancement in the thin films by having conductive regions within an insulating matrix.

In this article, we report on the modelling performed using Silvaco, Atlas device simulation, where we propose a new kind of enhancement mechanism within the $\mathrm{FE}$ process in hydrogenated amorphous silicon (a-Si:H). This model is equally applicable to all disordered and nano-crystalline materials. In particular, internal field enhancement where a conductive region is embedded in an insulating matrix. The simulation setup is shown in Figure 1 (a) with a $0.1 \mu \mathrm{m}$ thick a-Si:H film on a highly doped silicon substrate and a vacuum gap is $0.5 \mu \mathrm{m}$ for the unmodified control sample. Figure 1 (b) shows the setup where a conductive filament of $10 \mathrm{~nm}$ in diameter is incorporated into the bulk a$\mathrm{Si}: \mathrm{H}$ thin film.

Simulations were performed to investigate the role of "hot" electrons in the process of field emission from a-Si:H. A conductive filament is included in the thin film, and improvements in the emission current were observed in the 'hot' electron injection region of the current versus electric field data. This allows the required electric field for emission to be observed at around $8 \mathrm{~V} / \mu \mathrm{m}$. The mechanism that triggers such a 
phenomenon will be explained in term of a "worm-hole" effect observed in the internal electric field contained within the bulk a-Si:H thin film.

When examining the electric field lines and their strengths in the vicinity of the conductive filament, it is apparent that there is not only an electric field perpendicular to the filament, but also a high electric field parallel to the conductive filament. This lateral field acts as a "worm hole" to suck free electrons close to the filament towards it, which then get emitted to the positively biased anode via a transfer of momentum perpendicular to the electric field. We will support our preliminary data on simulation with experimental results.
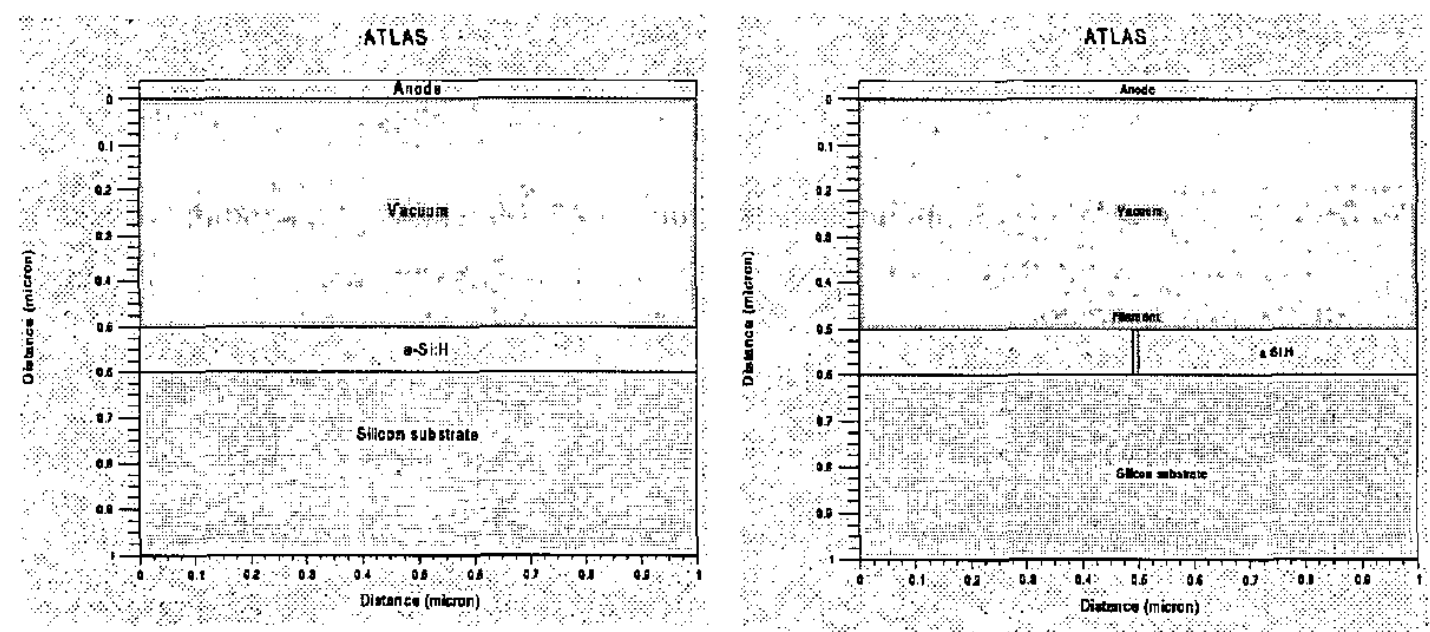

Figure 1. Simulation setup (a) without filament and (b) with filament

Dr. C H P Poa

Advanced Technology Institute, School of Electronics and Physical Sciences, University of Surrey, Guildford GU2 7XH, United Kingdom

Tel: 4414876099

Fax: 441483689404

E-mail: Patrick.poa@eim.surrey.ac.uk 